\title{
The nonlinear coupling of electromagnetic ion cyclotron and lower hybrid waves in the ring current region: the magnetic storm 1-7 May 1998
}

\author{
G. V. Khazanov ${ }^{1}$, E. N. Krivorutsky ${ }^{2}$, K. V. Gamayunov ${ }^{3}$, and L. A. Avanov ${ }^{1,4}$ \\ ${ }^{1}$ NASA, Marshall Space Flight Center, Huntsville, Alabama, USA \\ ${ }^{2}$ Center for Space Plasma \& Aeronomic Research, University of Alabama, Alabama, Huntsville, USA \\ ${ }^{3}$ Geophysical Institute, University of Alaska, Fairbanks, Alaska, USA \\ ${ }^{4}$ Space Research Institute of Russian Academy of Sciences, Moscow, Russia
}

Received: 11 June 2003 - Accepted: 30 July 2003 - Published: 14 April 2004

Part of Special Issue "International Workshops on Nonlinear Waves and Chaos in Space Plasmas"

\begin{abstract}
The excitation of lower hybrid waves (LHWs) is a widely discussed mechanism of interaction between plasma species in space, and is one of the unresolved questions of magnetospheric multi-ion plasmas. In this paper we present the morphology, dynamics, and level of LHW activity generated by electromagnetic ion cyclotron (EMIC) waves during the 2-7 May 1998 storm period on the global scale. The LHWs were calculated based on a newly developed selfconsistent model (Khazanov et. al., 2002) that couples the system of two kinetic equations: one equation describes the ring current (RC) ion dynamic, and another equation describes the evolution of EMIC waves. It is found that the LHWs are excited by helium ions due to their mass dependent drift in the electric field of EMIC waves. The level of LHW activity is calculated assuming that the induced scattering process is the main saturation mechanism for these waves. The calculated LHWs electric fields are consistent with the observational data.
\end{abstract}

\section{Introduction}

Wave-particle and wave-wave interactions are the crucial elements of magnetosphere and ionosphere plasma dynamics. Such interaction provides a channel of energy redistribution between different plasma populations, and leads to connection between physical processes developing on the different spatial and temporal scales. The low-hybrid waves are particularly interesting for plasma dynamics, because they couple well with both electrons and ions. Various mechanisms for LHW excitation have been studied as well as the phenomena produced by such waves (Davidson et al., 1977; Sonnerup,

Correspondence to: G. V. Khazanov

(george.khazanov@msfc.nasa.gov)
1980; Chang and Coppi, 1981; Bingham et al., 1984; Gurnett et al., 1984; Ganguli and Palmadesso, 1987; Pottelette et al., 1990; Omelchenko et al., 1994). In some cases, the LHW activity in magnetosphere has been observed simultaneously with low-frequency waves (LFWs) (LaBelle et al., 1988; Pottelette et al., 1990; McFadden et al., 1998). Such simultaneous wave activity also has been observed in active ionospheric sounding rocket experiments (Arnoldy, 1993; Bale, 1998). One reason for this may be that there is a common source for both waves. Another possible explanation is LHW generation due to the LFW activity (Khazanov et al., 1996, 1997a, b).

It is well known in the plasma physics that the LFWs with frequencies $\omega<\Omega_{i}$ (where $\Omega_{i}$ is the ion cyclotron frequency) could drive a host of high frequency waves through the drifts of the plasma particles produced by the former waves. LHW excitation is possible due to any transverse electric field with a frequency comparable to the ion cyclotron frequency, such as the fields of ion cyclotron, Alfvén, and fast magnetosonic waves. These waves are frequently observed in the ionosphere and plasmasphere (LaBelle et al., 1988; Pottelette et al., 1990; Erlandson et al., 1990; Fraser et al., 1992; Anderson et al., 1992). The study by Khazanov et al. (1997a) paid attention to the special role of heavy ions in this mechanism due to the fact that the particle drift velocity in the LFW electric field is mass dependent. They showed that the relative drift between the electrons and heavy ions across the ambient magnetic field (from the observed amplitudes of Alfvén waves) is sufficiently large to drive LHWs in magnetospheric plasma. The idea has been adopted to explain some observations in the space plasma (Khazanov et al., 1997b) and active ionospheric sounding rocket experiments (Bale et al., 1998).

In particular, this mechanism of LHW generation has been applied to the ring current region of the magnetosphere (Khazanov et al., 1997a, 2000). It is believed that the hot 
protons, which have large temperature anisotropy, are the source of Alfvén activity in that region (Kennel and Petschek, 1966). Therefore, the possible role of the LHW excitation due to the Alfvén activity for the energy exchange between the hot population and the core plasma, as well as for the Alfvén wave dynamics and saturation into the multicomponent plasma of ring current region, are a subject of interest. The studies of Khazanov et al. (1997a, 2000) were devoted to the investigation of this mechanism and analyses of the applicability of their results to the ring current region. They found that for the generation of LHWs the heavy ions drift velocity should be at least comparable with the thermal velocity of the light ions. This leads to the threshold for the LFW electric field, and the generation is possible only if this field exceeds the threshold. Therefore, it should be expected that the most favorable conditions for the proposed mechanism of LHW generation exist in the ring current region during the magnetic storms when this area is populated by heavy ions and the LF activity is high.

It should be noted that in the above-mentioned papers by Khazanov et al. (1997a, b, 2000), the LFW was assumed to be monochromatic, but usually the packets of Alfvén waves are observed (Olsen et al., 1987; Anderson et al., 1992). The wave energy in this case is redistributed in some frequency interval. Because the drift velocity depends on the electric field amplitude and frequency of the harmonics, the drift velocity and the threshold for the LFW electric field should be reconsidered. Verifying the applicability of their results to the ring current region Khazanov et al. (1997a, 2000) used the average wave and plasma parameters, or the observational data for some specific events. Therefore, these studies still did not answer the question what is the possible domain and duration of this phenomenon and its possible impact on the wave-plasma dynamics in the ring current region on the global scale.

In order to study the generation of LHWs on the global scale in the RC region, the core plasma and Alfvén waves parameters are needed that, at this point, could be found only on the base of global scale modeling. This study is based on the results from the self-consistent model of the magnetospheric RC and EMIC waves for the 2-7 May 1998 storm period (Khazanov et al., 2002, 2003). Below we present first the LFW activity and core plasma density distribution during this particular storm on the global scale. Then the drift of ions in the packets of EMIC waves is discussed. Next the dispersion equation for LHWs and the results of its solution on the global scale are presented and analyzed. In conclusion we estimate the possible level of LHW activity and the energy outflow from LHWs to the core plasma during the magnetic storm period.

\section{EMIC waves and core plasma characteristics}

The description of EMIC activity and core plasma density distribution for the 2-7 May 1998 storm period presented here is based on simulations described in detail by Khaz- anov et al., $(2002,2003)$. These results are calculated using the self-consistent RC model, which includes the drift kinetic equation for the RC energetic ions, and kinetic equation for EMIC waves in quasilinear approach. For particles, they are taking into account losses from the charge exchange, Coulomb collisions, ion-wave scattering, precipitation at low altitudes, and losses through the dayside magnetopause. Description of EMIC waves includes their generation by hot particles, absorption by the cold plasma, and reflection from ionosphere. Reflection index is frequency and normal angle dependent, and related to ionosphere parameters through $\mathrm{AE}$ index in these calculations. The changes in the vector of wave normal orientation along the magnetic field line, longitudinal and radial drifts of the wave packets are neglected. The dipole magnetic field and Kp dependent Volland-Stern convection field are adopted in these studies. The core plasma density is calculated using the threedimensional model of Angerami and Thomas (1964) adjusted to the time-dependent equatorial model of Rasmussen et al. (1993). Coulomb collisions of the RC ions with the thermal plasma are calculated for the multi-component core plasma model with the composition $77 \%$ of $\mathrm{H}^{+}, 20 \%$ of $\mathrm{He}^{+}$and $3 \%$ of $\mathrm{O}^{+}$. For all other processes the electronproton core plasma model is used. Core plasma temperature is assumed to be $1 \mathrm{eV}$. The boundary and initial conditions for the ring current ion distribution are based on the observational data from LANL, AMPTE/CCE and Explorer 45 satellites. To obtain the self-consistent initial conditions for EMIC waves the simulation was started using the background noise level of these waves (Akhiezer et al., 1975), and for the hot particles were used statistically derived quiet time ring current proton energy and pitch angle distributions (Sheldon and Hamilton, 1993; Garcia and Spjeldvik, 1985).

Figure 1 presents a history of the 2-7 May 1998 storm period in terms of EMIC wave magnetic field energy and core electron plasma densities. As can be seen from this figure the EMIC wave activity is essentially enhanced starting on 6 May, during the late recovery phase of this geomagnetic storm. It is consistent with the statistical observational data reported by Wentworth (1964). A detailed analysis of the wave activity zone morphology and dynamics was presented in papers (Khazanov et al., 2002, 2003). The maximum growth rate takes place for the EMIC waves with the wave vector directed along the ambient magnetic field line, and for the angles between these directions larger than $27^{\circ}$ the wave spectral energy density drops to the noise level. The growth rate is also negligible for the magnetic latitudes larger then $13^{\circ}$. Four shapes of the wave energy spectral density distribution can be identified from the modeling results (Khazanov et al., 2003). It is single peaked distributions: symmetric, right- and left-sides extended; and double-peaked ones. The last shape was found in $17 \%$ of analyzed waveforms and the peaks frequency separation is about $40 \%$ of the spectrum width. (More detailed description of the EMIC waves can be found in the paper by Khazanov et. al. (2003).) We will approximate all of them by Gaussian distribution instead of more accurate cubic splines (Khazanov et al., 2003). Such 

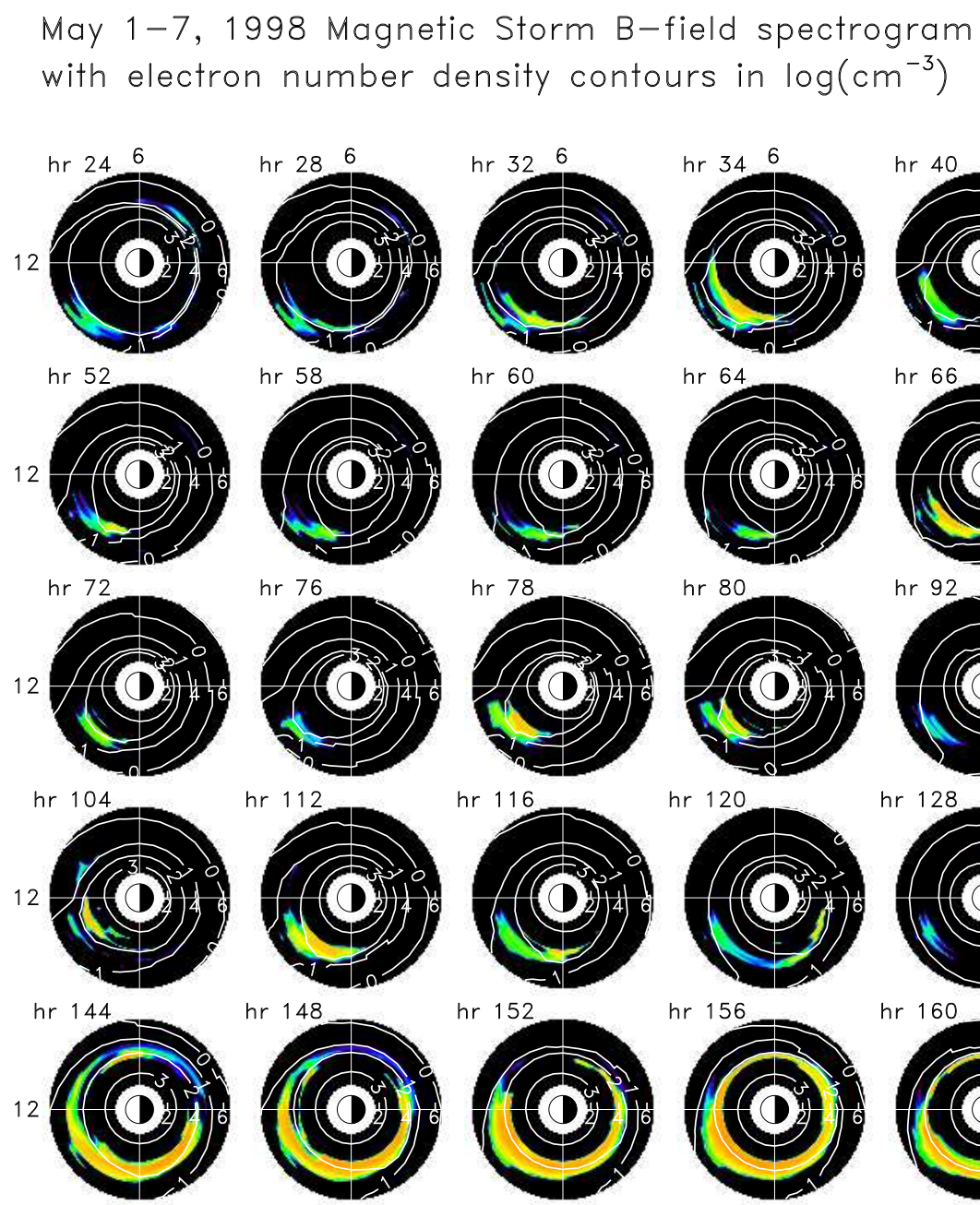

18
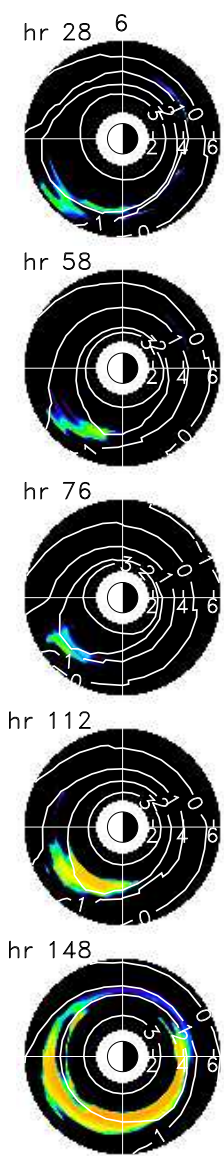

18
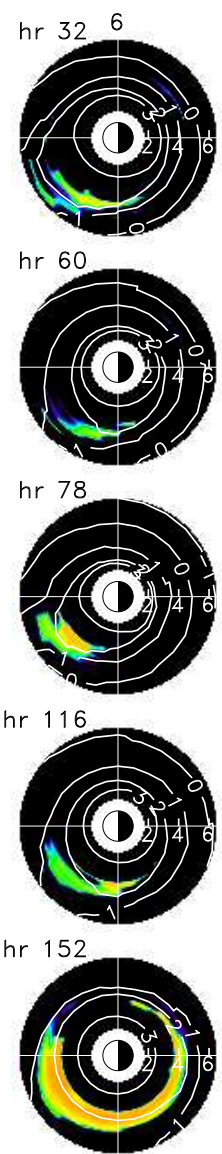

18
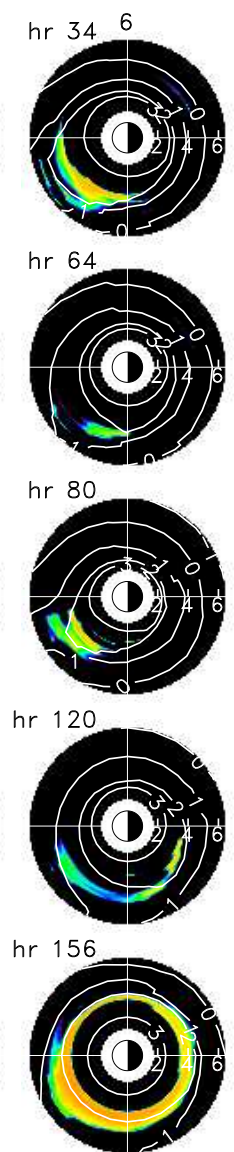

18
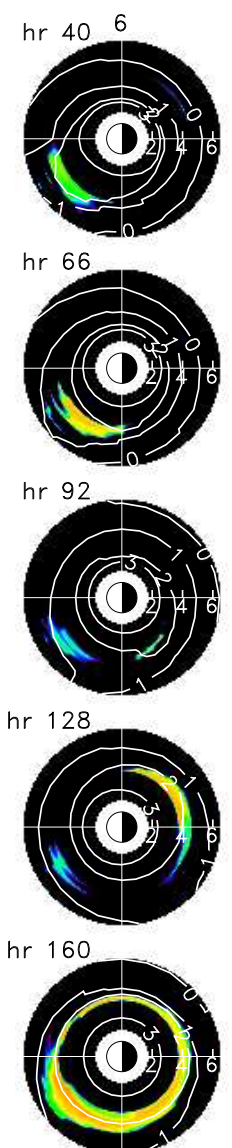

18
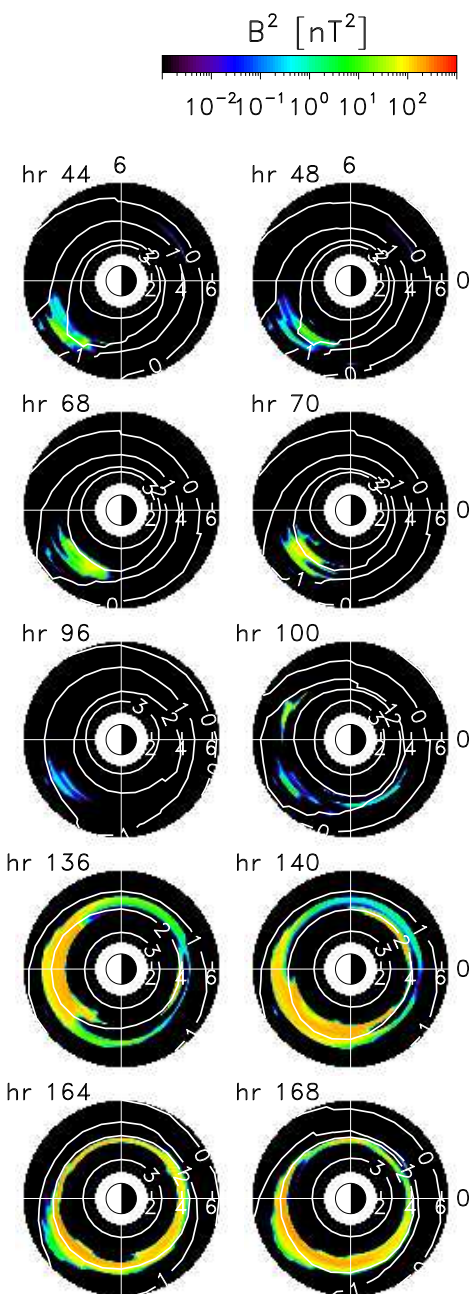

18

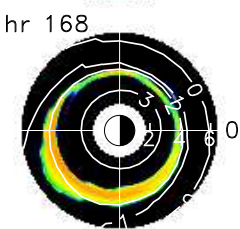

18

Fig. 1. Evolution of the squared EMIC wave magnetic field and core plasma density in (MLT, L-shell) - space during the 2-7 May 1998 storm period.

approximation overestimates the peak not more than at $20 \%$, and underestimates the width of the spectrum. Because the LHW generation depends on the particles drift velocity and therefore, proportional to the square root of the wave energy density the corresponding $10 \%$ overestimation will be neglected in the following analyses as well as the difference in the spectrum width. The last is not important because of the low field amplitudes at the wings of the spectrum. Therefore, we assume that the magnetic field energy spectral density of EMIC waves distribution is Gaussian:

$B_{v}^{2}=C \exp \left[-\frac{(v-\langle v\rangle)^{2}}{2 D^{2}}\right]=C \exp \left[-\frac{(1-x)^{2}}{2 d^{2}}\right]$

where: $B_{v}^{2}$ is the wave magnetic field energy spectral density; $v,\langle v\rangle$ are the current and mean frequencies, and $x=v /\langle v\rangle, d=D /\langle v\rangle$ are normalized frequency and the standard deviation. Khazanov et al. (2003) presented typical ex- amples for the four shapes of EMIC waves spectrums. The normalized standard deviations for these particular distributions are: $d=0.10,0.13,0.14$, and 0.19 . The corresponding ratios of the mean frequency to the hydrogen gyrofrequency $\langle v\rangle / \Omega_{H}$ are: $0.51,0.57,0.50$, and 0.55 .

\section{Generation of LHW}

Particles of plasma subjected to the LFW with the electric field perpendicular to the ambient magnetic field are drifting with the mass dependent velocities. The difference of these velocities can cause the beam instability in such a system, in particular the LHW generation. To calculate the particle drift velocity, the LF wave electric field is needed. Because the EMIC waves were calculated in quasilinear approximation, the energy density distribution of these waves is averaged over time, large in comparison with the wave period. This 


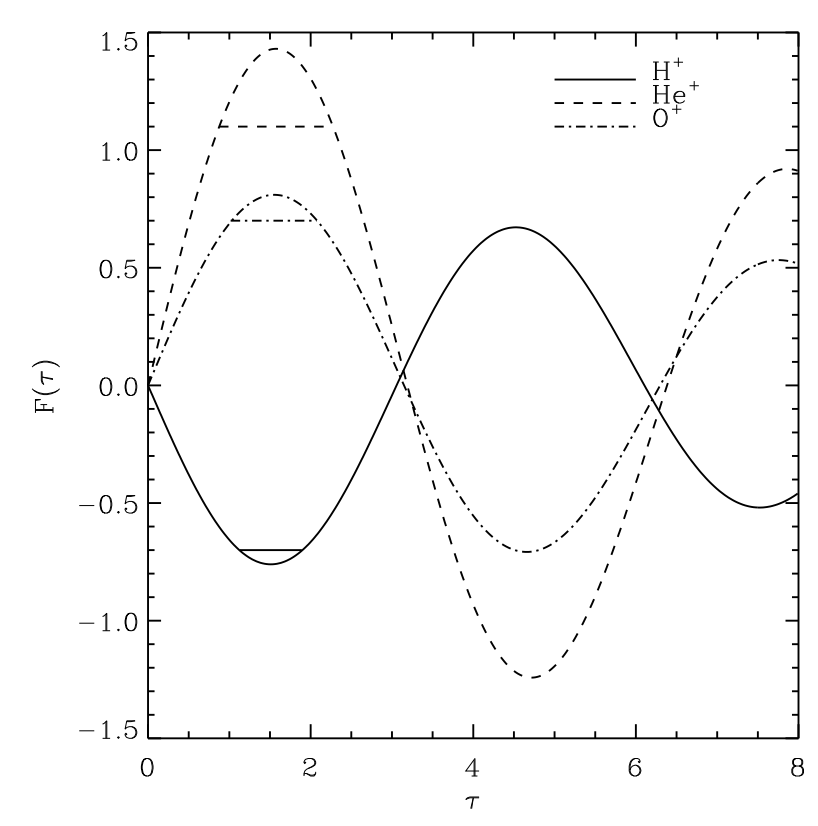

Fig. 2. Normalized ion drift velocity $u_{\alpha x} / \sqrt{2} v_{T H}=F(\tau) \sqrt{B^{2} / n}$ as a function on normalized time $\tau=t\langle v\rangle$.

time average is equivalent to the average over the statistical ensemble, where different realizations are defined by some set of phase, randomly changing from one realization to another. While the wave field exhibits randomness on the time scale large in comparison with the wave period, it is still regular on the time intervals smaller or around the period of the EMIC wave (Tsytovich, 1970). The frequency of the LHWs is about $\sqrt{\Omega_{H} \Omega_{e}}\left(\Omega_{H}, \Omega_{e}\right.$ are the hydrogen and electron gyrofrequencies respectively), and their period is much smaller than the EMIC waves period. We will restrict our analyses by such cases that the LHWs growth rate is also comparable to the frequency of EMIC waves and therefore, the time of LHWs excitation is comparable to the EMIC waves period. In fact, we will seek such cases that the LHW growth rate is at least 5 times greater than the EMIC wave frequency. Therefore, for such LH waves the characteristic time of generation is essentially smaller than the characteristic time at which the stochastic changes of EMIC waves takes place. Under this condition the EMIC wave field can be considered as regular for the drift velocity calculations. Based on the results presented in Sect. 2 we will assume that the EMIC wave is left-hand polarized, with the wave vector directed along the ambient magnetic field (axes $z$ ). Fourier components of the electric and magnetic fields of this wave are:

$$
\begin{aligned}
\boldsymbol{E}_{v} & =\frac{b_{v}}{N_{v}}\left(\boldsymbol{e}_{x}-i \boldsymbol{e}_{y}\right) e^{-i v t+i \kappa(v) z}, \\
\boldsymbol{B}_{v} & =i b_{v}\left(\boldsymbol{e}_{x}-i \boldsymbol{e}_{y}\right) e^{-i v t+i \kappa(v) z} \\
N_{v}^{2} & =1-\sum \frac{\omega_{p \alpha}^{2}}{v\left(v-\Omega_{\alpha}\right)}
\end{aligned}
$$

$$
\omega_{p \alpha}^{2}=\frac{4 \pi n_{\alpha} e^{2}}{m_{\alpha}}, \quad \Omega_{\alpha}=\frac{e_{\alpha} B}{m_{\alpha} c}, \alpha=e, \mathrm{H}^{+}, \mathrm{He}^{+}, \mathrm{O}^{+}
$$

Here $N_{v}^{2}$ is the plasma refractive index for the EMIC waves, and $n_{\alpha}$ are the plasma component densities. We also will assume, taking into account (1), that

$b_{v}=A \exp \left[-\frac{(v-\langle v\rangle)^{2}}{4 D^{2}}\right]$

where the coefficient $A$ is found from the condition:

$B^{2}=\left(\int_{-\infty}^{+\infty} b_{\nu} d v\right)^{2}$

and $B^{2}$ is the EMIC wave magnetic field energy density calculated from the numerical model presented in Fig. 1. Neglecting by the EMIC wave magnetic field, the relative velocity of an ion species $\alpha$, with respect to the electrons, can be calculated as (Khazanov et al., 1997a):

$\boldsymbol{u}_{\alpha}=\frac{e}{m_{H} \Omega_{H}} \int_{-\infty}^{+\infty} \frac{v \boldsymbol{E}_{v}}{\left(v-\Omega_{\alpha}\right)} d v$

In our calculations we used the electric field two times smaller than given by expression (2). Such field reduction according to the estimations essentially overlaps the errors related to the use of different approximations in numerical calculation of EMIC waves magnetic field energy density (Khazanov et al., 2002, 2003) and the calculation of corresponding electric fields and drift velocities in this paper. It can be found (after substitution in Eq. (5) of the EMIC electric field (2)) that the relative ion velocity for fixed plasma composition depends on parameters: $m_{H} / m_{\alpha},\langle v\rangle / \Omega_{H}, d$, and $B^{2} / n$. For a fixed point along the magnetic field line, $z=0$, the results of the velocity calculation for the hydrogen, helium, and oxygen ions are presented in Fig. 2. They are calculated for the Gaussian EMIC wave energy distribution with the normalized standard deviation $d=0.10$ and the mean frequency $\langle v\rangle=0.51 \Omega_{H}$. The figure presents the ions velocity due to an initially created set of waves with the initial energy density equal to the average density at a fixed point in space. Because the set of waves includes different frequencies, the ions velocity amplitude slowly diminishes. Such a slow rate of the amplitude change means that the field action on the ion is close to the action of a monochromatic wave. Calculation for the monochromatic wave with the mean frequency leads to the ion velocity amplitudes about $15 \%$ higher than presented in Fig. 2. For all four shapes of EMIC waves energy density distribution, when the Gaussian approximation of the shape is used, the relative ions velocities, calculated using the expression (5), are very close. The velocities dependence on the ambient magnetic field through the parameter $\langle v\rangle / \Omega_{H}$ (see expressions (3)-(5)) in the domain of interest, where the EMIC activity is high, is also weak. This permits us below to use the velocities averaged over the corresponding parameters $\langle v\rangle / \Omega_{H}, d$ with the accuracy 
May $1-7,1998$ Magnetic Storm

contours: $B^{2} / n=1.75 \quad B^{2} / n=2.6 \quad B^{2} / n=3.6 \quad B^{2} / n=5.0$
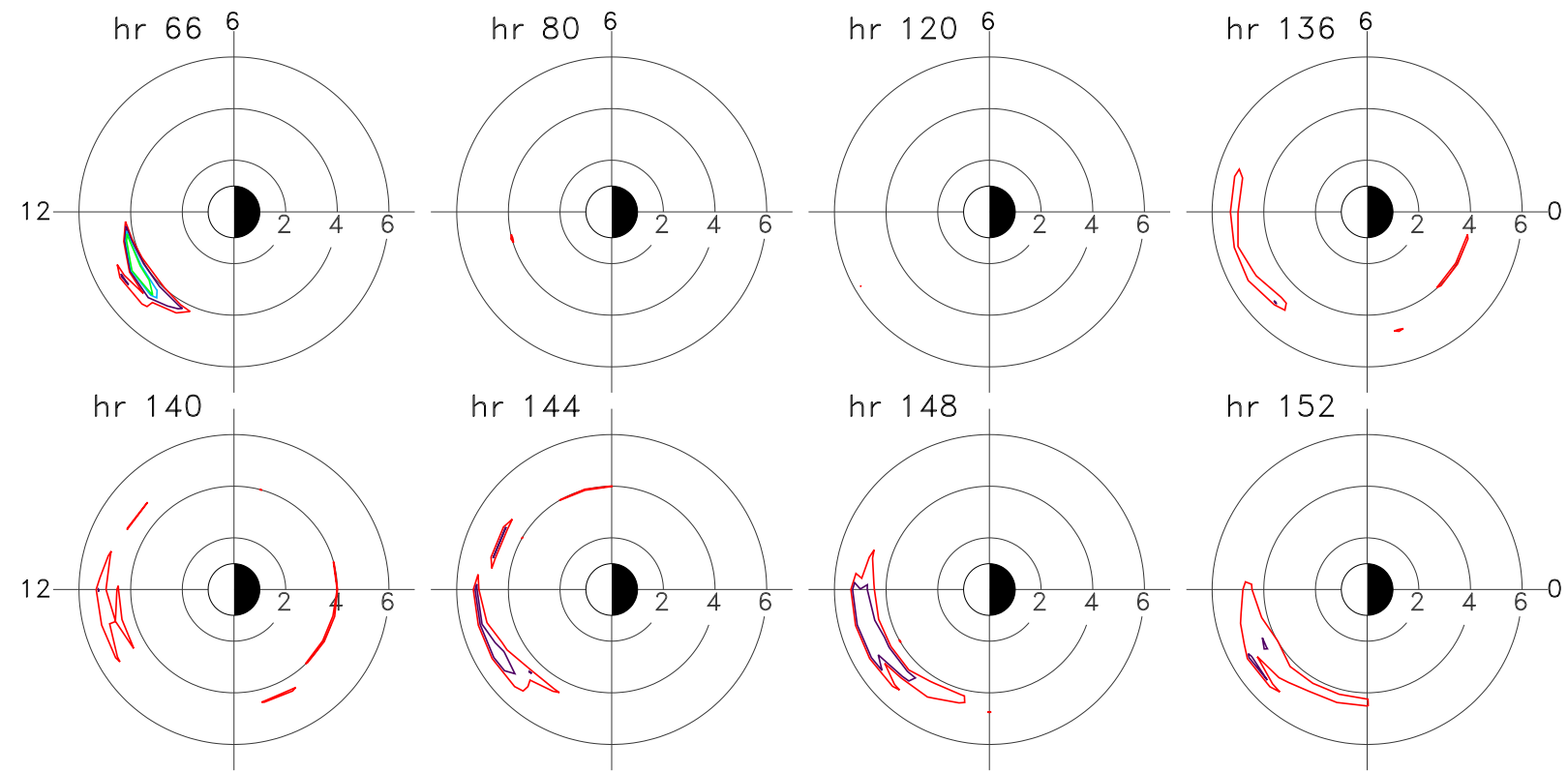

hr 152
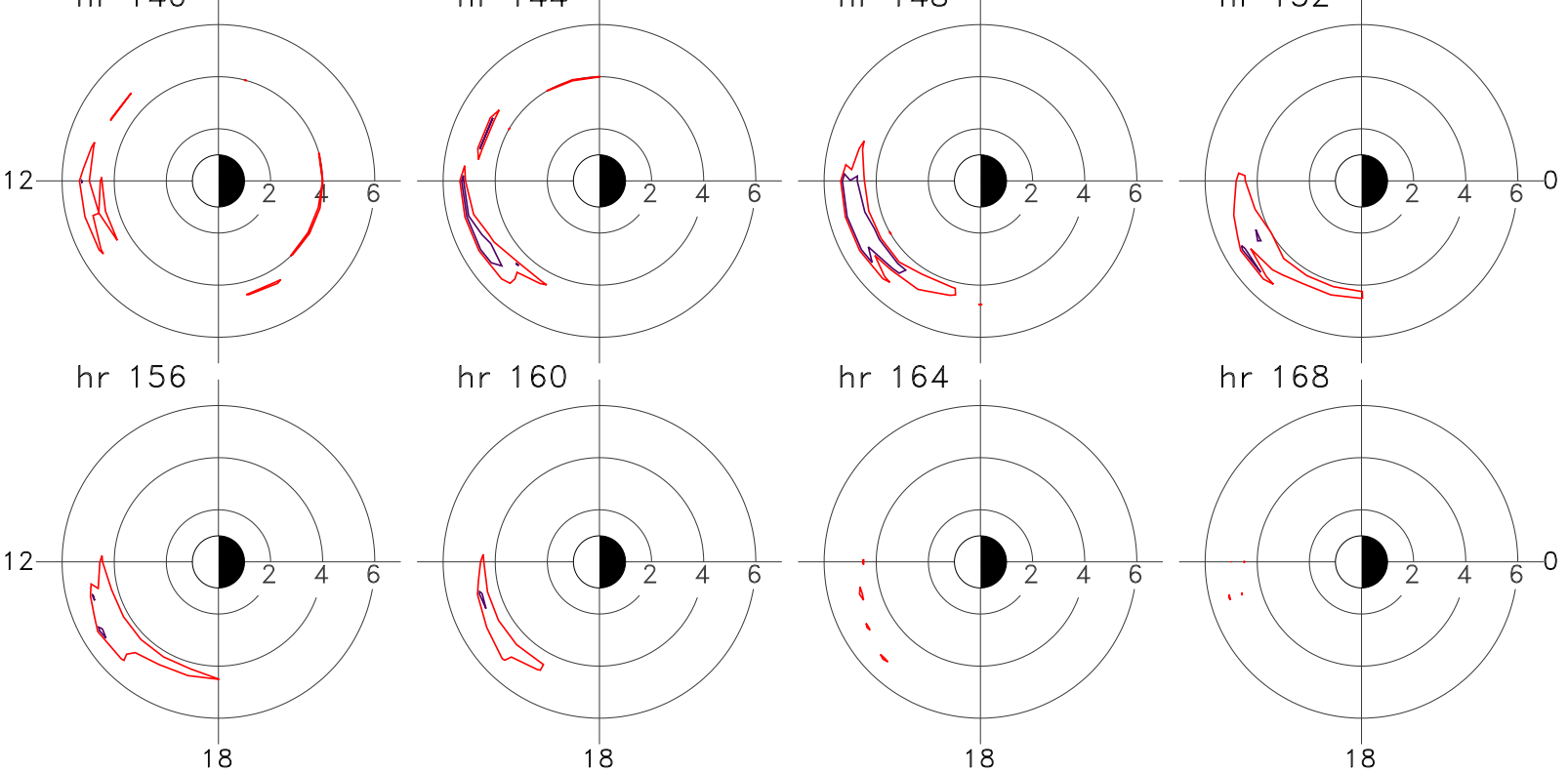

Fig. 3. Evolution of the squared EMIC wave electric field $\sim B^{2} / n$ (normalized) in (MLT, L-shell)-space during the 2-7 May 1998 storm period.

of about $15 \%$. The main dependence of the ion relative velocity is the dependence on the EMIC waves magnetic field energy and plasma densities and, as can be found from expression (5) has the form $u_{\alpha x} / \sqrt{2} v_{T H}=F(\tau) \sqrt{B^{2} / n}$, where the coefficient of proportionality, $F(\tau)$, presented by Fig. 2, is dependent on the $m_{H} / m_{\alpha}$ ratio. The dependence on $n$ for the drift velocity is because the electric field of EMIC waves is expressed with the help of the wave magnetic field and the phase velocity, which depends on the plasma density. These results, presenting the ion relative velocity with respect to the electrons, will be used to analyze the excitation of LHW in linear approximation (Akhiezer et al., 1975). The core plasma below is considered to be the multi-component, containing $77 \%$ of $\mathrm{H}^{+}, 20 \%$ of $\mathrm{He}^{+}$and $3 \%$ of $\mathrm{O}^{+}$. This thermal plasma composition is used for the calculation of the Coulomb collisions of the ring current ions in the numerical model (Khazanov et al., 2002, 2003).
It should be noted that a refined approach to the problem of LHW excitation by the field of the LF wave should be based on the weak turbulence theory, or the parametric approach, if we are neglecting by the finiteness of the LF wavelength (Gamayunov et al., 1992a). In this study we will use the approximate approach assuming that during the time of LHW generation the particles drift velocities can be considered as constant. We will also restrict the analyses by the case of unmagnetized ions. Then the dispersion equation for LHW has the following form (e.g. Akhiezer et al., 1975; Khazanov et al., 1997a):

$$
\begin{aligned}
1+\frac{\omega_{p e}^{2}}{k^{2} v_{T e}^{2}}\left[1+I_{0}(x) e^{-x} z_{e} Z\left(z_{e}\right)\right] \\
+\sum_{\alpha} \frac{\omega_{p \alpha}^{2}}{k^{2} v_{T \alpha}^{2}}\left[1+z_{\alpha} Z\left(z_{\alpha}\right)\right]=0
\end{aligned}
$$


$x=\frac{k_{\perp}^{2} v_{T e}^{2}}{\Omega_{e}^{2}} ; z_{e}=\frac{\omega}{\sqrt{2} k_{z} v_{T e}} ; z_{\alpha}=\frac{\omega-\boldsymbol{k}_{\perp} \boldsymbol{u}_{\alpha}}{\sqrt{2} k v_{T \alpha}} ;$

$k_{z}=k \cos \vartheta$

$v_{T}^{2}=\frac{T}{m} ; \omega_{p e}^{2}=\frac{4 \pi n e^{2}}{m_{e}} ; \omega_{p \alpha}^{2}=\frac{4 \pi n_{\alpha} e^{2}}{m_{\alpha}} ;$

$\alpha=\mathrm{H}^{+}, \mathrm{He}^{+}, \mathrm{O}^{+}$

where: $I_{0}(x)$ is the modified Bessel function; $Z\left(z_{\alpha}\right)$ is the plasma dispersion function; and $\omega$ and $\boldsymbol{k}$ are the frequency and the wave vector of the LH oscillations, respectively, and $\vartheta$ is the angle between the wave vector and the ambient magnetic field.

In order to obtain Eq. (6), we assumed that $\Omega_{\alpha} \ll \omega \ll \Omega_{e}$ and the motion of all ion species is unmagnetized, i.e. $k_{\perp}^{2} v_{T \alpha}^{2} / \Omega_{\alpha}^{2} \gg 1$. As follows from the analyses of Eq. (6), the LHWs, excited in our case, have wavelengths large in comparison with the Debye length, and the first term, the unit in Eq. (6), can be safely neglected. Equation (6) can then be split into two, for the real and imaginary parts as:

$$
\begin{aligned}
& 2+I_{0}(x) e^{-x} \operatorname{Re}\left[z_{e} Z\left(z_{e}\right)\right] \\
& \quad+\sum_{\alpha} c_{\alpha} \operatorname{Re}\left[1+z_{\alpha} Z\left(z_{\alpha}\right)\right]=0 \\
& I_{0}(x) e^{-x} \operatorname{Im}\left[z_{e} Z\left(z_{e}\right)\right] \\
& \quad+\sum_{\alpha} c_{\alpha} \operatorname{Im}\left[z_{\alpha} Z\left(z_{\alpha}\right)\right]=0
\end{aligned}
$$

where $c_{\alpha}$ is the concentration of ion species. Because of the Landau damping on electrons, only LHWs with the wave vector near normally directed to the ambient magnetic field can be excited. Therefore, the difference between $k$ and $k_{\perp}$ in $x$ and $z_{\alpha}$ can be neglected. Equations (7) are the equations for the real and imaginary parts of the normalized LHWs phase velocity $\left(\omega / \sqrt{2} k v_{T H}\right)$. For the fixed plasma composition the system of Eqs. (7) depends on three parameters: the ratio of the LHW length to the electron gyroradius $(x)$, the angle between the LHW vector and the ambient magnetic field $(\vartheta)$, and the ions velocity in the EMIC wave electric field $\left(u_{\alpha} / \sqrt{2} v_{T H}\right)$. This last dependence is in fact the dependence on the ratio of the magnetic field energy density of EMIC waves to the plasma density, $B^{2} / n$, as it was found from expression (5). Parameter $B^{2} / n$, which is plotted in Fig. 3, is calculated from the data presented in Fig. 1 on the global scale. For the local magnitude of this parameter we need to look for solutions to Eqs. (7), depending on the wavelength and the angle between the wave vector and the ambient magnetic field. Growing solutions to these equations exist only for ion velocities comparable to the hydrogen thermal velocity (Khazanov et al., 1997a). That means that the excitation of LHW can be expected only during a part of the EMIC wave period, when the ion drift velocity satisfies this condition. On the other hand, as it was stated above, the dispersion Eq. (6) is valid if, during a time of order $\gamma^{-1}$ (where $\gamma$ is the growth rate of LHW), the ion velocity can be considered as constant. To satisfy both of these conditions we will use such approach, solving the system (7). From Fig. 2 we will choose the normalized time interval $\Delta \tau= \pm 0.5, \quad(\tau=t\langle v\rangle)$, around the moment when the velocity is maximum, $\tau \approx 1.5$. During this time interval the velocity of ions are larger than the velocities at the ends of the interval, which are (see the Fig. 2) $u_{\mathrm{H} / \mathrm{O}}=(+/-) \times 0.7 \times v_{T H} \times \sqrt{2 B^{2} / n}$, $u_{\mathrm{He}}=-1.1 \times v_{T H} \times \sqrt{2 B^{2} / n}$ for hydrogen, oxygen and helium ( $B$ in nT, $n$ in $\mathrm{cm}^{-3}$ ). The choice of the time interval, $\Delta \tau= \pm 0.5$, is to some degree arbitrary, but it is restricted by two conditions named above: the velocity at the ends of the interval should be large enough compare to the hydrogen thermal velocity to drive the LHWs, and the time interval should be long enough compare to the LHW growth time. As a result of these restrictions, the permitted variations of the time interval are not large, and other possible choices of the time interval lead to the close results. These minimal velocities $\left(u_{\mathrm{H} / \mathrm{O}}=(+/-) \times 0.7 \times v_{T H} \times \sqrt{2 B^{2} / n}\right.$, $\left.u_{\mathrm{He}}=-1.1 \times v_{T H} \times \sqrt{2 B^{2} / n}\right)$ will be substituted in the system (7), and we will seek for such LH waves, that their period is at least 5 times smaller than the time interval $2 \Delta \tau$, and the characteristic growth time, $\gamma^{-1}$, is not larger than the same time interval. These restrictions, and the assumptions used to obtain the dispersion Eqs. (6) and (7), constitute the conditions that we imposed on the solutions of Eqs. (7):

$$
\begin{aligned}
& 10 \Omega_{H} \leq 0.1 \Omega_{e}, \quad 10 \frac{\Omega_{H}^{2}}{v_{T H}^{2}} \leq k^{2} \leq 0.1 \frac{\omega_{p H}^{2}}{v_{T H}^{2}}, \\
& \Delta t \equiv \frac{2 \Delta \tau}{\langle v\rangle} \geq \frac{10 \pi}{\omega}, \quad \Delta t \geq \frac{1}{\gamma}
\end{aligned}
$$

The first inequality here is imposed because we are looking for LHW. The second condition selects the wavelength larger then the Debye radius and smaller then the ion Larmor radius. The system of Eqs. (7) in the domain of parameters defined by inequalities (8) was solved by Broyden's method on the global scale. Results of these calculations are summarized in Table 1. This table presents the main characteristics of the LHW excitation process and the parameters of generated waves that are organized according to the magnitude of the parameter $B^{2} / n$.

The first column in this table characterizes the EMIC wave electric field as it was discussed above. The next three columns present the normalized drift velocities. The coincidence of hydrogen and oxygen drift velocity magnitudes (columns two and four) is accidental (Fig. 2), and holds on only with the presented accuracy. The next three columns describe the exited LH waves. From this data the wave frequency and wavelength for the listed angles of propagation can be calculated. The last column can be used for the growth rate calculation for a wave with a fixed frequency and wave vector.

The left hand and right hand quantities in the last three columns correspond one to another. They change from left to right close to monotonically, but not proportionally. As can be seen from this table, the larger the EMIC electric field the larger is the domain of the angles of LHW generation, $\vartheta$, their phase velocities, $\operatorname{Re} \omega / \sqrt{2} k v_{T H}$, and the maximum growth rate, $\operatorname{Im} \omega / \sqrt{2} k v_{T H}$. The angles of generation of the 
Table 1. Main characteristics of the LHWs excitation process.

\begin{tabular}{|c|c|c|c|c|c|c|c|}
\hline$B^{2} / n\left[\mathrm{nT}^{2} \mathrm{~cm}^{3}\right]$ & $\frac{u_{H}}{\sqrt{2} v_{T H}}$ & $\frac{u_{H E}}{\sqrt{2} v_{T H}}$ & $\frac{u_{0}}{\sqrt{2} v_{T H}}$ & $\cos \vartheta \sqrt{\frac{m_{H}}{m_{e}}}$ & $\frac{k^{2} v_{T e}^{2}}{\Omega_{e}^{2}}$ & $\frac{R e \omega}{\sqrt{2} k v_{T H}}$ & $\frac{\operatorname{Im} \omega}{\sqrt{2} k v_{T H}}$ \\
\hline \multirow[t]{2}{*}{1.75} & -0.8 & 1.4 & 0.8 & 0.03 & $0.07-0.11$ & $1.2-1.0$ & $0.20-0.16$ \\
\hline & & & & 0.3 & $0.1-0.16$ & $1.1-1.0$ & $0.17-0.13$ \\
\hline \multirow[t]{4}{*}{2.6} & -1.1 & 1.8 & 1.1 & 0.03 & $0.03-0.17$ & $1.4-1.0$ & $0.42-0.13$ \\
\hline & & & & 0.3 & $0.03-0.22$ & $1.5-1.0$ & $0.34-0.11$ \\
\hline & & & & 0.6 & $0.07-0.25$ & $1.6-1.2$ & $0.21-0.10$ \\
\hline & & & & 0.7 & $0.15-0.17$ & $1.5-1.4$ & 0.13 \\
\hline \multirow[t]{3}{*}{3.6} & -1.3 & 2.1 & 1.3 & 0.03 & $0.02-0.13$ & $1.6-1.1$ & $0.61-0.15$ \\
\hline & & & & 0.3 & $0.02-0.17$ & $1.8-1.1$ & $0.50-0.13$ \\
\hline & & & & 0.8 & $0.07-0.23$ & $1.9-1.6$ & $0.20-0.11$ \\
\hline \multirow[t]{4}{*}{5.3} & -1.6 & 2.5 & 1.6 & 0.03 & $0.02-0.08$ & $1.9-1.2$ & $0.80-0.20$ \\
\hline & & & & 0.3 & $0.02-0.11$ & $2.1-1.3$ & $0.72-0.16$ \\
\hline & & & & 0.9 & $0.08-0.23$ & $2.2-1.9$ & $0.31-0.11$ \\
\hline & & & & 1.1 & $0.10-0.21$ & $2.3-2.1$ & $0.16-0.11$ \\
\hline
\end{tabular}

LHWs are restricted by Landau damping on the electrons and the waves are generated nearly perpendicular to the ambient magnetic field. The phase velocity is always smaller than the helium drift velocity. That is the relative drift of helium to electrons that drives the instability. The drift of oxygen ions leads to the amplification of shorter waves (the drift velocity is larger than the phase velocity), but oxygen absorbs the longer ones (opposite ratio between these two velocities). In our case the input of oxygen to the LHW generation is small, due to its small content in plasma (3\%). It can be found from the dispersion Eqs. (7) and the data in the Table 1 that the hydrogen and helium density perturbations in the LHW are of the opposite signs and partially compensated. The generated wavelengths, $\lambda$, are in the range of $10 \rho_{e} \leq \lambda \leq 40 \rho_{e}$ (where $\rho_{e}$ is the electron gyroradius), and for the larger wavelength the phase velocity is larger. From the side of shorter waves $\left(\lambda<10 \rho_{e}\right)$ damping prevail on the excitation. Longer waves have smaller frequencies, i.e. larger periods. Therefore, our condition that the time interval, during which the drift velocity drops below the chosen level, should be larger than 5 wave periods and larger than the growth character time, restricts the wavelength from the larger side (8). The growth rate, $\operatorname{Im} \omega / \sqrt{2} k v_{T H}$, is restricted from the smaller side by the last time condition from inequalities (8). It is a growing function of the EMIC electric field $\left(\sqrt{B^{2} / n}\right)$; this function is larger for the wave vectors normal to the ambient magnetic field, and the ratio $\operatorname{Im} \omega / \operatorname{Re} \omega$ changes in the range $0.05 \div 0.4$. The character of the LHW excitation described above is similar to that obtained for the LHWs, excited by an ion beam propagating across the magnetic field and forming a jet spectrum in $k$-space (Musher et al., 1986). With the results presented in the Table 1 , combined with the distribution of the parameter $B^{2} / n$ from the Fig. 3 , the LHW excitation on the global scale can be analyzed. As it is seen from this figure and the table, the LHWs are mainly excited at the end of the storm period, when the EMIC activity is well developed (Fig. 1). The periods of LHWs excitation during the storm directly reflect the dependence on the EMIC wave electric field, which is proportional to the $\sqrt{B^{2} / n}$ ratio. That is why the LHW excitation takes place, for example, at $66 \mathrm{~h}$, but not at $34 \mathrm{~h}$ (Figs. 1 and 4). The EMIC waves magnetic fields are close, but the density is approximately 10 times larger at $34 \mathrm{~h}$. As a result, the electric field and, therefore the drift velocities, are about three times smaller in this case. The EMIC waves electric field, with good accuracy, can be calculated with the help of expressions (2)-(4) as $E[\mathrm{mV} / \mathrm{m}]=280 \sqrt{B^{2} / L^{3} n}$ and in the region of interest is 3-6 mV/m, as can be seen from Fig. 1 .

\section{Discussion and Conclusion}

As presented above, the LHW growth rate permits us to identify the regions where the LHW activity can be expected during the storm evolution (Table 1, Fig. 3). Using the calculated LHW growth rates we can also estimate what is the expected level of LHW activity. We will assume that the level of the thermal noise in plasma is the initial level of LH activity. This level is apparently surpassed during the storm and such a choice is a guarantee from an overestimation in what follows. The level of thermal noises in Maxwellian protonelectron plasma can by found in Akhiezer et al. (1975). We will use this result for our estimations in spite of the $20 \%$ admixture of helium. Because the initial level of LHW energy density is the multiple before the exponent in the expression for the wave energy density at later moments,

$E^{2}(t)=E_{\text {thermal }}^{2}(t=0) e^{2 t \operatorname{Im} \omega}$ 
May $1-7,1998$ Magnetic Storm
$E[\mathrm{mV} / \mathrm{m}]$ spectrograms for LHW
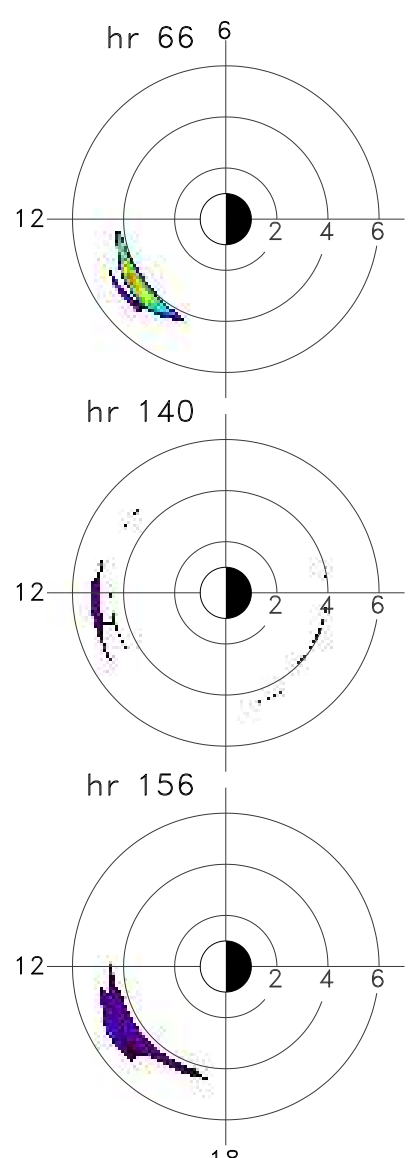

18

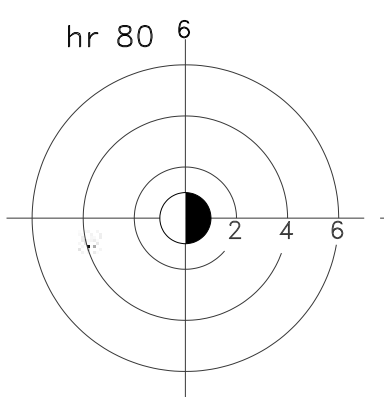

hr 144
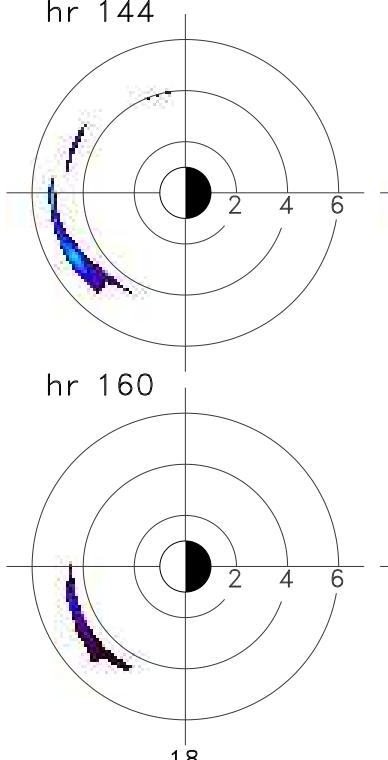

18

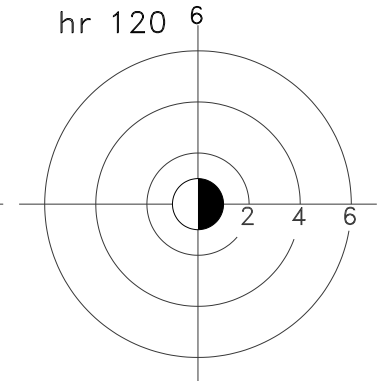

hr 148
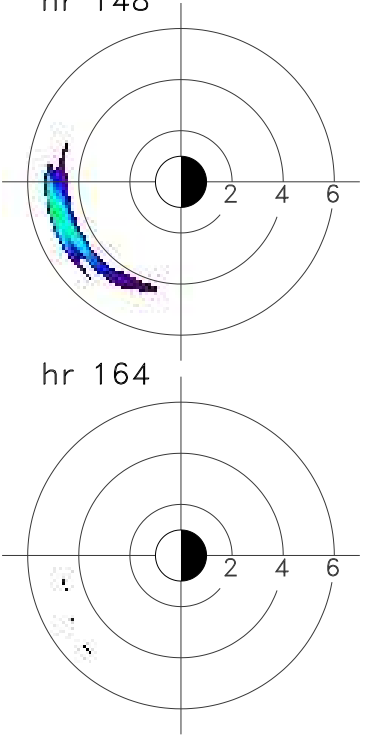

18
$\mathrm{E}[\mathrm{mV} / \mathrm{m}]$

$\begin{array}{lllll}0.20 & 0.30 & 0.40 & 0.50 & 0.60\end{array}$

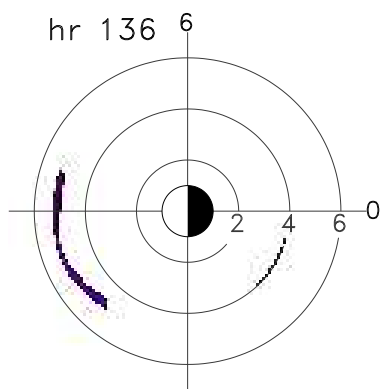

hr 152
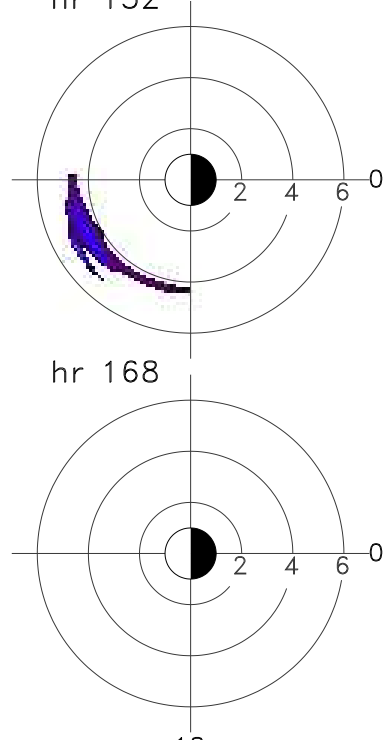

18

Fig. 4. Low-hybrid wave electric field in (MLT, L-shell)-space during the 2-7 May 1998 storm period.

this discrepancy (plasma in our case is multicomponent), as well as the use of average parameters below, should not change the estimations essentially. The spectral component of the electric field space correlation function is:

$\left\langle E^{2}\right\rangle_{k}=8 \pi^{2} T \frac{\Omega_{e}^{2}}{\Omega_{e}^{2}+\omega_{p e}^{2}}$

Calculating the volumes of the $k-$ space corresponding to the different drift velocities determined by the parameter $\sqrt{B^{2} / n}$ (Table 1), and multiplying this volume on the spectral density (10), the initial LHW energy densities, $E^{2}(t=0)$, are found to be:

$$
\begin{array}{lcccc}
\sqrt{B^{2} / n}\left[\mathrm{nT}^{2} \mathrm{~cm}^{3}\right] & 1.75 & 2.60 & 3.60 & 5.30 \\
10^{12} E^{2}(t=0)\left[\mathrm{mV}^{2} / \mathrm{m}^{2}\right] & 2.3 & 4.0 & 5.3 & 6.60
\end{array}
$$

As can be seen from Eq. (11) the initial energy levels are close for our set of drift velocities. Because the LHW spectral energy density (10) is independent on the wave vector, it also follows from Eq. (11) that the volumes of $k$-space, in which the LHW is generated, are growing with the energy density of EMIC wave electric field. Now we are able to calculate the LHW energy density at the end of the time interval $2 \Delta \tau=1$, where $\tau=t\langle v\rangle$ is the time normalized on the average EMIC wave period. Recall that during this time the drift ions velocities exceed their values used in the LHW growth rate calculations and, therefore, at list during this time the generation of LHWs hold on. To perform the calculations with the help of expression (9) we need also the growth rate. The growth rates are $k$-dependent as can be seen from the Table 1 . The region of LHW activity in $k$-space forms a jet as we have seen above. Approximately, it is a cone with the axis normal to the ambient magnetic field. The main part of the cone volume is located near the outer boundary, $k=k_{\max }$. As it was found in calculations the growth rate sharply drops in the narrow vicinity near $k_{\max }$ (about $10 \%$ of $k_{\max }$ ) but remains close to the constant for the essential part of $k$-region before this drop. Thus, the growth rate from this main region 
was used to calculate the LHW energy density and electric field at the end of the time interval $2 \Delta \tau \equiv t\langle\nu\rangle$ from Eq. (9). The results are:

$$
\begin{array}{lcccc}
\sqrt{B^{2} / n}\left[\mathrm{nT}^{2} \mathrm{~cm}^{3}\right] & 1.75 & 2.60 & 3.60 & 5.30 \\
E(t=1 /\langle v\rangle)\left[\mathrm{mV}^{2} / \mathrm{m}^{2}\right] & 0.002 & 0.06 & 0.20 & 0.60 .
\end{array}
$$

To calculate these LHW electric fields we neglected by their non-linear damping, which can restrict the growth and saturate the LHW activity on the lower level. As it was found by Musher et al. (1986) for LHWs excited by an ion beam propagating across a magnetic field in electron-proton plasma, the main mechanism, which leads to the saturation of such waves, is induced scattering by ions and electrons. The case, when the beam is formed by a heavy component of plasma due to electric field of a low frequency wave, was studied by Khazanov et al. (1997a). They came to the same conclusion; i.e. quasilinear diffusion only slightly diminishes the LHW growth rate and the saturation results from the induced scattering. Therefore, the quasilinear effects will not change the results (12) dramatically. Following Musher et al (1978) the damping rate for induced scattering can be presented as follows:

$\gamma^{s c}=\frac{\omega_{p e}^{4}}{\omega\left(\Omega_{e}^{2}+\omega_{p e}^{2}\right)} \frac{E^{2}}{8 \pi n T}$

For a quasi-stationary state the growth rate of the LHW from the Table 1 should be equal to this damping rate. From this condition we can calculate the saturation levels of LHW activity for different values of the $B^{2} / n$, i.e. for different drift velocities. This saturation level for the LHW electric field is in the range $1-2 \mathrm{mV} / \mathrm{m}$, and exceeds the electric field for all four cases presented in Eq. (12). Therefore, at the end of the time period $2 \Delta \tau \equiv\langle v\rangle t=1$ the level of LHW electric fields presented in Eq. (12) can be expected. After this the EMIC wave electric field and the helium drift velocity drop below the level needed for the LHW generation and the LHW activity will be damp during the remaining part of the EMIC wave period. Then the generation will be switched on again if the EMIC waves are still present in this domain. What the resulting quasi-stationary level of LHWs will be, if the EMIC activity exists long enough in the region, depends first of all on the damping rate during the remaining part of the EMIC wave period, when the ions drift velocity is below the level needed for LHW generation. To estimate the LHW damping and following growth at the next period of EMIC wave, we can look on the problem as two initial value problems. Then at the end of one part of the EMIC waves period we have the initial LHW characteristics for the next part of the period. For initial perturbation with a fixed wave vector $\boldsymbol{k}$ the electric field can be found as (Aleksandrov et al., 1984):

$E(t, \kappa)=-i \int \frac{D(t=0, \kappa)}{\omega \varepsilon(\omega, \kappa)} e^{-i \omega t} d \omega \sim C e^{-i \omega(\kappa) t}$

Here: $D((t=0, \kappa)$ is the initial electric displacement, $\varepsilon(\omega, k)$ is the dielectric permittivity, and $\omega(k)$ is the solution of the dispersion equation $\varepsilon(\omega, k)=0$ for the LHW mode in our case. As can be seen from expression (14) the evolution of the LHW electric field for the harmonic with fixed $k$ depends on the $\operatorname{Im} \omega(k)$. Therefore, we need also the damping rates for the wave vectors of the excited LHW activity, which are presented in the Table 1 . We calculated these damping rates and the total growth and damping during one EMIC wave period, $2 \pi /\langle\nu\rangle$, i.e.:

$$
\Gamma=\left.\delta t \operatorname{Im} \omega(k)\right|_{\text {growth }}+\left.\left(\frac{2 \pi}{\langle v\rangle}-\delta t\right) \operatorname{Im} \omega(k)\right|_{\text {damping }}
$$

where $\delta t=2 \Delta \tau /\langle\nu\rangle$. It was found that for all cases when the LHWs are excited, there are always some wave vectors $\boldsymbol{k}$ for which the LHW activity is enriched during the EMIC wave period. The existence of such $\boldsymbol{k}$ can be understood from the data presented in the Table 1 for the growth rate and the $k$-depends of the damping. The growth rate is largest for small $k$ and decreases for the larger $k$. The damping for waves with small $k$ is small and increases for larger $k$. Therefore, some harmonics with small $k$ can survive during the damping part of the EMIC wave period, if the angle $\vartheta$ (Table 1) is such, that the Landau damping for electrons is not too strong. Surviving LH harmonics form a narrow jet in $k$-space and we can neglect by the difference between the different harmonics in this jet. Note, that the expression (13) for the scattering rate depends on the total energy density of the LHW electric field in the used approximation. If the EMIC wave acts long enough then the exact level of the LHW perturbations that survives the damping part of the period are not important for our estimation. These perturbations will grow at least until the induced scattering will not stop this process on some quasi-stationary level. To calculate this level, we can introduce using Eq. (15) an effective growth rate as the growth rate averaged over the EMIC wave period for the most quickly growing harmonic:

$\langle\gamma\rangle_{\mathrm{eff}}=\frac{\langle\nu\rangle}{2 \pi} \Gamma$

Equating the growth rate (16) to the damping rate due to induced scattering (13) we found the LHW electric field for the quasi-stationary state

$$
\begin{array}{lcccc}
\sqrt{B^{2} / n}\left[\mathrm{nT}^{2} \mathrm{~cm}^{3}\right] & 1.75 & 2.60 & 3.60 & 5.30 \\
E[\mathrm{mV} / \mathrm{m}] & 0.20 & 0.30 & 0.55 & 0.65
\end{array}
$$

We compared these electric fields to the threshold of the modulational instability of the LHW (Musher et al., 1978), which in our case can be presented as follow:

$$
\frac{E_{t r}^{2}}{8 \pi n T}=\frac{m_{e}}{m_{H}} \frac{k^{2} v_{T e}^{2}}{\omega_{p e}^{2}}
$$

This threshold is higher than the electric fields given by (17), but in the last case $(0.65 \mathrm{mV} / \mathrm{m})$ the threshold is $1.1 \mathrm{mV} / \mathrm{m}$. Apparently the modulational instability can be excited in some points during the storm period. Therefore, depending 
on the time during which the EMIC waves exists in the region of LHW generation the LHW electric fields in the range between listed in (12) and (17) can be reached.

We have no observational results for the LHW during this storm period, but we still can compare our results with the data obtained from other events. Data from the 6 May 1982 event measured by the Dynamics Explorer 1 satellite gave the experimental value of LHW electric field $0.003 \mathrm{mV} / \mathrm{m}$. (Olsen et al., 1987). Another case of LHW activity was observed by AMPTE IRM satellite at 6 April 1985, when the LHW electric field was $0.6 \mathrm{mV} / \mathrm{m}$ (LaBelle et al., 1988). These data are in the range of electric fields values listed above in (12) and (17) and testify that the calculated results are reasonable.

We also estimated the outflow of the energy from LHW to the core plasma. There are two main channels of energy outflow from the LHW. One of them is the induced scattering on ions and electrons. Because the frequency of LHWs is low the energy is mainly transformed to the electrons (Musher et al., 1978). For the stationary case the energy flow, $Q$, can be estimated from the balance between the energy pumped to the LHWs by the helium drift and the energy outflow from this waves to the particles. It follows from this balance (Khazanov et al., 1997a) that:

$$
\frac{Q}{n T} \sim \frac{\gamma^{2} \omega}{\Omega_{e}^{2}}
$$

We used for these estimations the effective growth rate (16). For two last cases presented in (17) the energy of particles doubles during an hour. For weaker electric fields the effect is small. Another channel of particle energization is due to the quasilinear effects. Mainly, the energy is directed to the hydrogen. The admixture of oxygen ions is small (3\%), as well as their thermal velocities, compared to the phase velocity of the LHWs and this channel of energy outflow is negligible. Because the waves are generated in the narrow cone around the normal to the ambient magnetic field, the quasilinear effects for electrons are also small. Details of these calculations can be found in Khazanov et al. (1997a). We estimated this effect for the case that the LHW electric field is $0.55 \mathrm{mV} / \mathrm{m}$ with the help of expressions (23) and (32) from this reference. Kinetic energy gained by hydrogen ion is approximately $K(t) \sim 0.1 K(t=0) t^{0.4}$ and doubles during an hour. Because the phase wave velocity in this case is only about two times larger than the hydrogen thermal velocity the portion of particles involved in this interaction is large enough and constitutes $\sim 10 \%$ of the hydrogen population. Results presented above characterize on the global scale the morphology, dynamics and level of LHW activity generated by EMIC waves during the 2-7 May 1998 storm period. The latter waves were calculated in the framework of the selfconsistent model for the ring current particles and generated by these particles EMIC waves. As the input to the models involved in these calculations the observational data were used. Drift velocities of $\mathrm{H}^{+}, \mathrm{He}^{+}$, and $\mathrm{O}^{+}$were then found, and the region of LHW generation on the global scale was calculated. Assuming induced scattering as the saturation mechanism for the LHWs, their quasi-stationary level is estimated. In these estimations for the processes of induced scattering we used the electron-proton model. Because of different approximations involved in our calculations we two times reduced the electric field of the EMIC waves in the study of LHW generation. Analyses of this precaution lead us to conclusion that the region of LHW generation and the level of such activity are apparently underestimated in this study. Obtained under these approximations results for the LHWs electric field are consistent with the observational data. It should be stressed that to determine the role of the discussed mechanism of LHW generation for the ring current region and separate it from other sources of LH activity, detailed analyses of events based on the measurements of EMIC and LH waves, as well as cold plasma parameters, is required. To eliminate the restrictions that have been used to calculate the drift velocity and support our conclusions regarding the non-linear stage of LHW evolution, direct modeling PIC simulations are needed.

Edited by: G. S. Lakhina

Reviewed by: two referees

\section{References}

Aleksandrov, A. F., Bogdankevich, L. S., Rukhadze, A. A.: Principles of Plasma Electrodynamics, Berlin, New York, SpringerVerlag, 1984.

Anderson, B. J., Erlandson, R. E., and Zanetti, L. J.: A statistical study of Pc 1-2 magnetic pulsations in the equatorial magnetosphere: 1. Equatorial occurrence distributions, J. Geophys. Res., 97, 3075-3088, 1992.

Akhiezer, A. I.: Plasma electrodynamics, vol. 2, Pergamon Press, New York, 1975.

Angerami, J. J. and Thomas, J. O.: Studies of planetary atmospheres, 1, The distribution of ions and electrons in the Earth's exosphere, J. Geophys. Res., 69, 4537-4560, 1964.

Arnoldy, R. L.: Transverse ion acceleration by active experiments, Auroral Plasma Dynamics, edited by Lysak, R. L., AGU, Washington, D. C., Geophys. Monogr. Ser., vol. 80, 195-202, 1993.

Bale, S. D., Kellogg, P. J., Erickson, K. N., Monson, S. J., and Arnoldy, R. L.: Ponderomotive lower hybrid wave growth in electric fields associated with electron beam injection and transverse ion acceleration, Adv. Space Res., 21 735-21 741, 1998.

Bingham, R., Bryant, D. A., and Hall, D. S.: A wave model for the aurora, Geophys. Res. Lett., 11, 327-330, 1984.

Chang, T. and Coppi, B.: Lower hybrid acceleration and ion evolution in the subauroral region, Geophys. Res. Lett., 8, 1253-1256, 1981.

Davidson, R. C., Gladd, N. T., Wu, C. S., and Huba, J. D.: Effects of finite plasma beta on the lower-hybrid-drift instability, Phys. Fluids, 20, 301-312, 1977.

Erlandson, R. E., Zanetti, L. J., Potemra, T. A., Block, L. P., and Holmgren, G.: Viking magnetic and electric field observations of Pc 1 waves at high latitudes, J. Geophys. Res., 95, 5941-5955, 1990.

Fraser, B. J., Samson, J. C., Hu, Y. D., McPherron, R. C., and Russel, C. T.: Electromagnetic ion cyclotron waves observed near 
the oxygen cyclotron frequency by ISEE 1 and 2, J. Geophys. Res., 97, 3063-3074, 1992.

Gamayunov, K. V., Krivorutsky, E. N., Veryaev, A. A., and Khazanov, G. V.: Parametricexcitation of longitudinal oscillations by a lower frequency pump wave, Plasma Physics and Controlled Fusion, V34, \#7, 1359-1367, 1992a.

Gamayunov, K. V., Krivorutsky, E. N., Veryaev, A. A., and Khazanov, G. V.: Saturation of Alfvén oscillations in the ring current region due to generation of lower hybrid waves, Planet. Space Sci., 40, 477, 1992b.

Ganguli, S. B. and Palmadesso, P. J.: Plasma transport in the auroral return current region, J. Geophys. Res., 92, 8673-8690, 1987.

Garcia, H. A. and Spjeldvik, W. N.: Anisotropiy characteristics of geomagnetically trapped ions, J. Geophys. Res., 90, 347-358, 1985.

Gurnett, D. A., Huff, R. L., Menietti, J. D., Burch, J. L., Winningham, J. D., and Shawhan, S. D.: Correlated low-frequency electric and magnetic noise along the auroral field lines, J. Geophys. Res., 89, 8971-8985, 1984.

Kennel, C. F. and Petschek, H. E.: Limit on stably trapped particle fluxes, J. Geophys. Res., 71, 1-28, 1966.

Khazanov, G. V., Gamayunov, K. V., Jordanova, V. K., and Krivorutsky, E. N.: A self-consistent model of the interacting ring current ions with electromagnetic ICWs. Initial results: Waves and precipitating fluxes, J. of Geophys. Res., 107, 6, 10.1029/2001JA000180, 2002.

Khazanov, G. V., Gamayunov, K. V., and Jordanova, V. K.: Selfconsistent model of magnetospheric ring current and electromagnetic ion cyclotron waves: The 2-7 May 1998, storm, J. of Geophys. Res., submitted, 2003.

Khazanov, G. V., Gamayunov, K. V., and Liemohn, M. W.: Alfvén waves as a source of lower-hybrid activity in the ring current region, J. Geophys. Res., 105, 5403-5409, 2000.

Khazanov, G. V., Krivorutsky, E. N., Moore, T. E., Liemohn, M. W., and Horwitz, J. L.: Lower hybrid oscillations in multicomponent space plasmas subjected to ion cyclotron waves, J. Geophys. Res., 102, 175-184, 1997a.

Khazanov, G. V., Krivorutsky, E. N., Liemohn, M. W., and Horwitz, J. L.: A model of lower hybrid wave excitation compared with observetions by Viking, Geophys. Res. Lett., 24, 23992402, 1997b.

Khazanov, G. V., Moore, T. E., Krivorutsky, E. N., Horwitz, J. L., and Liemohn, M. W.: Lower hybrid turbulence and ponderomotive force effects in space plasmas subjected for large-amplitude low-frequency waves, Geophys. Res. Lett., 23, 797-800, 1996.
LaBelle, J., Treumann, R. A., Baumjohann, W., Haerendel, G., Sckopke, N., Paschmann, G., and Lühr,: The duskside plasmapause/ring current interface: Convection and plasma wave observations, J. Geophys. Res., 93, 2573-2590, 1988.

McFadden, J. P., Carlson, C. W., Ergun, R. E., Chaston, C. C., Mozer, F. S., Temerin, M., Klumpar, D. M., Shelley, E. G., Peterson, W. K., Moebius, E., Kistler, L., Elphic, R., Strangeway, R., Cattell, C., and Pfaff, R.: Electron modulation and ion cyclotron waves observed by FAST, Geophys. Res. Lett., 25, 2045-2048, 1998.

Musher, S. L., Rubenchik, A. M., and Shapiro, I. Ya.: Nonlinear effects in the propagation of an ion beam across a magnetic field, Sov. Phys. JETP, 63, 519-528, 1986.

Musher, S. L., Rubenchik, A. M., and Sturmn, B. I.: Collective effects associated with low hybrid heating of plasma, Plasma Phys. Sov., 20, 1131-1139, 1978.

Olsen, R. C., Shawhan, S. D., Gallagher, D. L., Green, J. L., Chappell, C. R., and Anderson, R. R.: Plasma observations at the Earth's magnetic equator, J. Geophys. Res., 92, 2385-2407, 1987.

Omelchenko, Yu. A., Shapiro, V. D., Shevchenko, V. I., AshourAbdalla, M., and Schriver, D.: Modified lower hybrid fan instability excited by precipitating auroral electrons, J. Geophys. Res., 99, 5965-5976, 1994.

Pottelette, R., Malingre, M., Dubouloz, N., Aparicio, B., Lundin, R., Holmgeen, G., and Marklund, G.: High-frequency waves in the Cusp/Cleft regions, J. Geophys. Res., 95, 5957, 1990.

Rasmussen, C. E., Guiter, S. M., and Thomas, S. G.: Twodimensional model of the plasmasphere: Refilling time constants, Planet. Space Sci., 41, 35-43, 1993.

Sheldon, R. B. and Hamilton, D. C.: Ion transport and loss in the Earth's quiet ring current, 1, Data and standard model, J. Geophys. Res., 98, 13 491-13 508, 1993.

Sonnerup, B. U. O.: Theory of the low latitude boundary layer, J. Geophys. Res., 85, 2017, 1980.

Tsytovich, V. N.: Nonlinear Effects in Plasma, Plenum, New York, 1970.

Wentworth, R. C.: Enhancement of Hydromagnetic emissions after geomagnetic storms, J. Geophys. Res., 69, 2291-2298, 1964. 